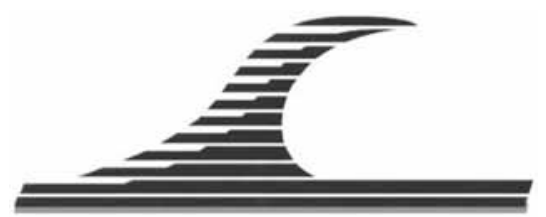

Revue Paralia, Volume 4 (2011) pp 2.1-2.12

Mots-clés : Vague, Houle, Canal à houle numérique, Interactions fluide-structure, Systèmes Récupérateurs

d'Energie des Vagues (SREV) C Editions Paralia CFL

\title{
Modélisation non-linéaire des interactions des vagues avec un corps mobile immergé
}

\author{
Etienne GUERBER ${ }^{1}$, Michel BENOIT ${ }^{1}$, Stephan GRILLI ${ }^{2}$, Clément BUVAT $^{1}$ \\ 1. Laboratoire d'Hydraulique Saint-Venant, Université Paris-Est, \\ (unité de recherche commune EDF R\&D, CETMEF, Ecole des Ponts ParisTech), \\ 6 quai Watier, 78400 Chatou, France. \\ etienne.guerber@edf.fr ; michel.benoit@edf.fr ; clement.buvat@edf.fr \\ 2. Department of Ocean Engineering, University of Rhode Island, \\ Narragansett, RI 02882, USA. \\ grilli@oce.uri.edu
}

\section{Résumé :}

Les travaux présentés concernent le développement d'un code de simulation avancé permettant de décrire le mouvement d'un corps immergé sous l'action des vagues, avec des mouvements de grande amplitude. A terme, cet outil est destiné à modéliser le comportement de certains types de Systèmes Récupérateurs d'Energie des Vagues (SREV) immergés.

Dans cette étude on adopte une approche potentielle pour la partie hydrodynamique, dans un cadre 2DV (i.e. dans un plan vertical), correspondant au cas d'un canal à houle numérique. Le modèle utilisé pour la génération et la propagation des vagues est un modèle potentiel complètement non-linéaire, fondé sur une méthode d'éléments de frontières d'ordre élevé, développé par Grilli et ses collaborateurs depuis une vingtaine d'années. Ce modèle, déjà largement validé sur différents types d'applications océaniques et côtières, a été modifié pour prendre en compte la présence d'un corps rigide immergé, fixe ou mobile, avec le calcul des efforts de pression hydrodynamique s'exerçant sur le corps. Une méthodologie spécifique a été développée pour résoudre le problème couplé hydrodynamique/mécanique. Nous présentons deux cas de validation pour lesquels les résultats du modèle numérique sont comparés à des résultats d'autres modèles mathématiques : théorie analytique de WU (1993) sur le cas d'un cylindre en mouvement circulaire imposé et théorie linéaire d'EVANS et al. (1979) sur le cas d'un cylindre soumis à des forces externes (forces de rappel, force représentant l'extraction d'énergie des vagues,...), en plus des efforts hydrodynamiques.

Soumis le 30 novembre 2010, accepté le 9 février 2011, en ligne le 21 mars 2011.

La seule version examinée est celle écrite en français. La ou les autres versions n'étant pas examinées par le comité de rédaction de la revue, sont donc publiées sous l'entière responsabilité du ou des auteurs.

\section{A TRANSLATED VERSION IN ENGLISH IS AVAILABLE ONLINE}

Pour citer cet article :

GUERBER E., BENOIT M., GRILLI S., BUVAT C. (2011). Modélisation non-linéaire des interactions des vagues avec un corps mobile immergé. Revue Paralia, Vol. 4, pp 2.1-2.12.

DOI:10.5150/revue-paralia.2011.002

(disponible en ligne - http://www.paralia.fr - available online) 


\section{Introduction}

Les énergies renouvelables marines connaissent un fort regain d'intérêt ces dernières années, et en particulier l'énergie houlomotrice, avec la mise au point et les tests de plusieurs SREV. Parmi les différentes technologies proposées, les SREV de type "absorbeur ponctuel" utilisent le mouvement oscillant de corps flottants ou immergés sous l'effet des vagues. Ces corps sont souvent immergés à une faible profondeur sous la surface de l'eau et décrivent des mouvements de grande amplitude (voir par exemple le système CETO, MANN et al., 2007). C'est pourquoi les modèles fondés sur l'hypothèse de mouvements de faible amplitude et/ou l'hypothèse d'une surface libre linéarisée ne sont pas adaptés à la simulation du système couplé hydrodynamiquedynamique du corps. Les travaux présentés ici consistent à développer un modèle numérique capable de calculer les interactions non-linéaires vagues/corps en 2 dimensions d'espace (2DV), pour être ensuite appliqué à la modélisation de la dynamique des SREV dans des états de mer réels (vagues irrégulières).

Une première étape dans ce projet consiste à résoudre le problème non-linéaire couplé pour le cas d'un cylindre horizontal immergé. Nous présentons ci-après les principales caractéristiques du modèle numérique développé ainsi que deux cas de validation pour un cylindre de section circulaire immergé, d'abord en mouvement imposé et ensuite en mouvement "libre" sous l'effet de plusieurs forces.

De nombreux auteurs se sont intéressés au calcul des interactions non-linéaires entre les vagues et un corps cylindrique. Ainsi CHAPLIN (1984) a effectué une série de tests en canal à vagues et a mesuré en particulier l'influence du nombre de Keulegan-Carpenter $\left(K_{C}\right)$ sur les efforts hydrodynamiques exercés sur le corps. Plus récemment, WU (1993) a adopté une approche analytique pour calculer les efforts exercés sur un cylindre en mouvement forcé de grande amplitude, en utilisant des conditions de surface libre linéarisées et des conditions exactes sur le corps. Les travaux de ces deux auteurs ont été largement utilisés pour valider un ensemble de modèles numériques tels que le modèle Sindbad de COINTE (1989) fondé sur une méthode d'éléments de frontières (BEM : Boundary Element Method), ou encore le modèle développé par KENT \& CHOI (2007) utilisant une méthode spectrale d'ordre élevé (HOS : High-Order Spectral method).

\section{Formulation mathématique}

\subsection{Présentation générale du modèle}

Nous utilisons un canal à houle numérique (NWT : Numerical Wave Tank) fondé sur un modèle potentiel complètement non-linéaire (FNPF : Fully Nonlinear Potential Flow) appliqué au calcul des interactions non-linéaires (forces hydrodynamiques et mouvement induit) entre l'écoulement dû aux vagues et un corps immergé représentant une section d'un SREV ancré sur le fond marin. Il s'agit d'une extension du modèle développé au cours des 20 dernières années par Stephan Grilli et ses collaborateurs 
(GRILLI \& SUBRAMANYA, 1996 ; GRILLI, 1997 ; GRILLI \& HORRILLO, 1997). Les équations du modèle sont rapidement présentées dans la suite (pour plus de détails, se reporter aux références ci-avant). Le potentiel des vitesses $\phi(\underline{x}, t)$ est utilisé pour décrire un écoulement supposé irrotationnel et incompressible dans le plan $(x, z)$, et le champ de vitesses est désigné par $\underline{u}=\nabla \phi=(u, w)$.

L'équation de continuité sur le domaine fermé fluide $\Omega(t)$, de frontière $\Gamma(t)$, est une équation de Laplace sur le potentiel (voir Fig.1 pour un schéma du domaine de calcul) : $\nabla^{2} \phi=0 \quad \operatorname{sur} \Omega(t)$

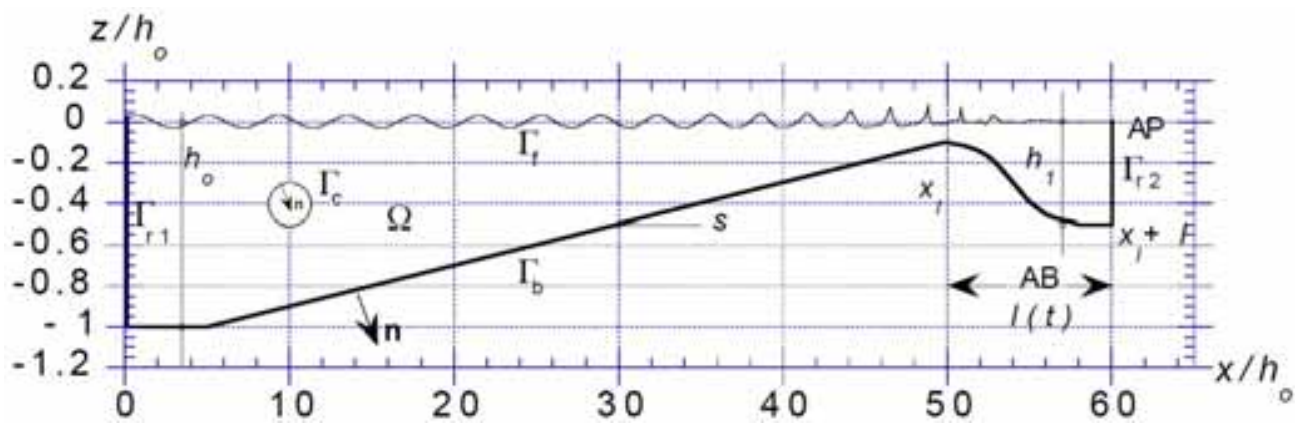

Figure 1. Exemple de domaine de calcul $\Omega(t)$ et frontières associées.

A la surface libre $\Gamma_{f}(t)$, le potentiel vérifie les conditions cinématique et dynamique de surface libre,

$$
\left\{\begin{array}{l}
\frac{D \underline{r}}{D t}=\left(\frac{\partial}{\partial t}+\underline{u} \nabla\right) \underline{r}=\underline{u}=\nabla \phi \\
\frac{D \phi}{D t}=-g z+\frac{1}{2} \nabla \phi \cdot \nabla \phi+\frac{p_{a}}{\rho}
\end{array} \quad \text { sur } \Gamma_{f}(t)\right.
$$

respectivement, avec $\underline{r}$ le vecteur position de la surface libre, $g$ l'accélération de la gravité, $p_{\mathrm{a}}$ la pression atmosphérique et $\rho$ la masse volumique du fluide. $\mathrm{Au}$ fond, supposé stationnaire, une condition de non-pénétration est imposée :

$$
\frac{\partial \phi}{\partial n}=\nabla \phi \cdot \underline{n}=0 \quad \text { sur } \Gamma_{b}
$$

$\underline{n}$ désigne le vecteur normal à la frontière considérée, orienté vers l'extérieur du domaine fluide.

Sur la frontière gauche du domaine $\Gamma_{r 1}(t)$, des vagues périodiques ou irrégulières peuvent être générées par le mouvement d'un batteur plan de type "volet" ou "piston". Il est aussi possible d'utiliser une méthode de génération exacte de vagues périodiques fondée sur la théorie de la fonction de courant. A l'extrémité droite du domaine, une plage absorbante $(A B)$ est implémentée pour réduire la réflexion des vagues sur la frontière $\Gamma_{r 2}(t)$. Plus de détails sur la génération et l'absorption des vagues dans le modèle sont disponibles dans GRILLI \& HORRILLO (1997). 
Sur le corps immergé $\Gamma_{c}(t)$, une condition spécifique est imposée, détaillée dans la partie suivante.

L'équation (1) est transformée en une équation intégrale sur les frontières (BIE) à l'aide de la seconde identité de Green; elle est ensuite résolue par la méthode des éléments de frontières. L'équation intégrale est évaluée en $\mathrm{N}$ nœuds de discrétisation sur les frontières et $\mathrm{M}$ éléments d'ordre élevé sont définis pour interpoler les variables entre les nœuds de discrétisation. Dans la suite, des éléments quadratiques isoparamétriques sont utilisés sur les frontières latérales et sur le fond. Des éléments linéaires isoparamétriques sont disposés sur le corps et des éléments cubiques sont utilisés sur la surface libre pour assurer la continuité de la pente de la frontière entre chaque élément. Sur ces derniers éléments, plus connus sous le nom d'éléments d'interpolation cubique mixte, la géométrie est modélisée par des fonctions de forme cubique et les variables physiques sont interpolées entre chaque paire de nœuds à l'aide d'un élément isoparamétrique "glissant" à 4 nœuds. Les expressions des intégrales sur les éléments (régulières, singulières et quasi-singulières) sont données dans GRILLI \& SUBRAMANYA (1996).

Les conditions de surface libre (2) sont intégrées en temps grâce à un développement en séries de Taylor au second ordre exprimées en fonction du pas de temps $\Delta t$ et des dérivées temporelles lagrangiennes de $\phi$ et $r$. Les termes du second ordre sont exprimés en dérivant de façon lagrangienne les équations (2) et calculés en résolvant une seconde BIE portant sur $\left(\partial \phi / \partial t, \partial^{2} \phi / \partial t \partial n\right)$ dont les conditions aux limites sont formulées à partir de la solution de la première BIE. Les expressions détaillées des séries de Taylor sont données dans GRILLI (1997).

Ce canal à houle numérique a été modifié pour prendre en compte la présence d'un corps rigide complètement immergé sous la surface libre. Deux situations sont envisagées : (i) le cas d'un corps en mouvement forcé (qui comprend le cas du corps fixe); et (ii) le cas du corps en mouvement "libre" (sous l'effet de divers efforts).

\subsection{Corps en mouvement forcé}

Lorsque le corps décrit un mouvement imposé, on impose à sa frontière une condition de Neumann sur le flux normal du potentiel :

$$
\frac{\partial \phi}{\partial n}=\underline{\dot{\alpha}} \cdot \underline{n} \quad \text { sur } \Gamma_{c}(t)
$$

où $\underline{\dot{\alpha}}$ désigne le vecteur vitesse des nœuds à la frontière du corps, connu dans le cas d'un mouvement imposé. Puisqu'un second problème de Laplace est résolu pour $\left(\partial \phi / \partial t, \partial^{2} \phi / \partial t \partial n\right)$, une condition supplémentaire doit être imposée pour calculer $\partial \phi / \partial t$. A l'instar de COINTE (1989), VAN DAALEN (1993) et TANIZAWA (1995), une condition de Neumann portant sur $\partial^{2} \phi / \partial t \partial n$ est imposée à la frontière du corps :

$$
\frac{\partial^{2} \phi}{\partial t \partial n}=\underline{\ddot{\alpha}} \cdot \underline{n}+\dot{\theta}\left(\underline{\dot{\alpha}} \cdot \underline{s}-\frac{\partial \phi}{\partial s}\right)-\left(\frac{1}{R} \frac{\partial \phi}{\partial s}+\frac{\partial^{2} \phi}{\partial s \partial n}\right) \dot{\alpha} \cdot \underline{s}+\left(\frac{\partial^{2} \phi}{\partial s^{2}}-\frac{1}{R} \frac{\partial \phi}{\partial n}\right) \underline{\dot{\alpha}} \cdot \underline{n} \operatorname{sur} \Gamma_{c}(t)
$$


$\underline{\alpha}$ et $\dot{\theta}$ sont respectivement l'accélération solide du point à la frontière du corps et la vitesse de rotation du corps. $1 / R$ est la courbure locale de la frontière, et $\underline{s}$ et $\underline{n}$ sont les vecteurs localement normal et tangent à $\Gamma_{c}(t)$. Les équations (4) et (5) sont semblables aux conditions imposées pour la génération de vagues par un batteur plan (GRILLI \& HORRILLO, 1997 ; GRILLI, 1997).

\subsection{Corps en mouvement "libre"}

Lorsqu'aucun mouvement du corps n'est prescrit, on parle de corps en mouvement "libre". Dans ce cas, l'accélération $\underline{\ddot{\alpha}}$ est une inconnue du problème et l'équation (5) ne peut être utilisée comme condition de Neumann pour résoudre le problème sur $\partial \phi / \partial t$. De plus, la vitesse $\underline{\alpha}$ dans l'équation (4) doit être calculée en tant que solution du problème couplé fluide-structure.

Si l'on considère un corps quelconque rigide de masse $M$ et de moment d'inertie $I$ par rapport à son centre d'inertie, les équations du mouvement s'écrivent :

$\left\{\begin{array}{l}M \underline{\ddot{x}}=\oint_{\Gamma_{c}} p \underline{n} d \Gamma_{c}+M \underline{g}+\underline{F}_{e x t} \\ I \ddot{\theta} \underline{e}_{y}=-\oint_{\Gamma_{c}} p(\underline{r} \times \underline{n}) d \Gamma_{c}+\underline{M}_{e x t}\end{array}\right.$

$\underline{\ddot{x}}$ désigne l'accélération du centre d'inertie, $\ddot{\theta}$ l'accélération angulaire du solide par rapport au centre d'inertie, $\underline{F}_{\text {ext }}$ est un terme pouvant représenter des efforts extérieurs agissant sur la structure (ancrage, effort d'extraction de l'énergie des vagues, etc.), $\underline{r}$ est le vecteur position du point d'intégration par rapport au centre d'inertie et $\underline{e}_{y}$ est le vecteur normal au plan $(x, z)$, défini par $\underline{e}_{y}=\underline{e}_{z} \times \underline{e}_{x}$. Enfin la pression $p$ agissant sur le corps est donnée par la relation (non-linéaire) de Bernoulli :

$p=-\rho\left(\frac{\partial \phi}{\partial t}+\frac{1}{2} \nabla \phi \cdot \nabla \phi+g z\right)$

Le calcul de la pression est rendu délicat par le fait que le terme $\partial \phi / \partial t$ est inconnu le long de $\Gamma_{c}(t)$. Par ailleurs, dans le problème de Laplace sur $\partial \phi / \partial t$, la condition de Neumann (5) sur le corps est également inconnue. Plusieurs auteurs ont proposé des méthodes pour surmonter cette difficulté : (i) une méthode de décomposition des modes (VINJE \& BREVIG, 1981), (ii) une méthode itérative (SEN, 1993 ; CAO et al., 1994), (iii) une méthode indirecte (WU \& EATOCK-TAYLOR, 1996) et (iv) une méthode implicite (VAN DAALEN, 1993 ; TANIZAWA, 1995). Cette dernière a été choisie pour notre modèle car elle ne nécessite aucune sous-itération et aucun potentiel "artificiel" n'est à introduire. Cette méthode utilise les équations (5), (6) et (7) pour en déduire une nouvelle équation intégrale, sous la forme :

$\frac{\partial^{2} \phi}{\partial t \partial n}(\underline{x})+\oint_{\Gamma_{c}} K(\underline{x}, \underline{\xi}) \frac{\partial \phi}{\partial t} d \Gamma_{c}(\underline{\xi})=\gamma(\underline{x})$

Cette équation est alors discrétisée par la méthode des éléments de frontière ; la matrice $K$ est régulière, symétrique et ne dépend que de la géométrie du corps. La fonction $\gamma$ est 
également calculée explicitement à chaque itération. Après avoir résolu le premier problème de Laplace sur le potentiel, l'équation (8) est ajoutée au système matriciel du problème sur $\partial \phi / \partial t$ de façon à avoir autant d'équations que d'inconnues. Ce second système matriciel est résolu par une méthode de décomposition LU basée sur la technique de Khaletski. Après avoir calculé la pression (Eq. 7), les équations du mouvement (Eq. 6) sont intégrées en temps et fournissent la position et la vitesse du corps au pas de temps suivant. Plusieurs schémas d'intégration ont été comparés sur des cas simples d'oscillateurs mécaniques avec amortissement, et le schéma de Newmark a été retenu :

$$
\left\{\begin{array}{l}
\dot{\underline{x}}_{n+1}=\underline{\dot{x}}_{n}+\Delta t\left((1-\gamma) \ddot{\ddot{x}}_{n}+\gamma \underline{\ddot{x}}_{n+1}\right) \\
\underline{x}_{n+1}=\underline{x}_{n}+\Delta t \underline{\dot{x}}_{n}+\Delta t^{2}\left(\left(\frac{1}{2}-\beta\right) \underline{\ddot{x}}_{n}+\beta \underline{\ddot{x}}_{n+1}\right)
\end{array}\right.
$$

Le choix s'est porté sur les paramètres $\gamma=1 / 2$ et $\beta=1 / 4$ correspondant à la méthode dite de l'accélération moyenne, ce qui en fait un schéma d'ordre 2 en temps et inconditionnellement stable, au moins pour les systèmes à dynamique linéaire. Etant donné que ce schéma est implicite ( $\underline{\ddot{x}}_{n+1}$ est inconnue en début de pas de temps), des itérations sont nécessaires. Nous avons utilisé comme valeur initiale une extrapolation polynomiale en temps à partir des valeurs des quantités inconnues aux cinq pas de temps précédents. Avec ce choix, quelques itérations seulement sont nécessaires pour converger (i.e. 1 à 3 itérations, dans nos simulations).

En pratique, le pas de temps $\Delta t$ est imposé par le solveur hydrodynamique et mis à jour à chaque itération en fonction d'un nombre de Courant optimal $C_{0}($ de l'ordre de 0,45$)$ et de la distance minimale entre deux nœuds voisins de la surface libre $\Delta r_{\min }$ :

$\Delta t=C_{0} \frac{\Delta r_{\min }}{\sqrt{g d}}$

où $d$ est la profondeur d'eau locale. Plus d'informations sur la stabilité et la convergence du schéma en temps utilisé pour le solveur hydrodynamique sont données dans GRILLI (1997).

\section{Cas du cylindre en mouvement imposé}

Le problème de radiation créée par les mouvements de grande amplitude d'un cylindre immergé dans un fluide infini à surface libre initialement au repos a été traité de façon analytique par WU (1993). Ce dernier n'effectue aucune approximation sur la condition au niveau du corps, mais utilise des conditions de surface libre linéarisées. Wu formule la solution sur le potentiel à l'aide d'un développement en multipôles sphériques généralisés et calcule les efforts hydrodynamiques exercés sur un cylindre de rayon $R$ en mouvement de rotation circulaire dans le sens horaire et d'orbite de rayon $C$. Il considère un seul nombre d'onde $k R=0,5$ et 8 orbites adimensionnées $C / R$. Le nombre d'onde des vagues générées par le cylindre est relié à la fréquence du mouvement 
circulaire par la relation de dispersion linéaire en profondeur infinie $k=\omega^{2} / g$. Wu démontre qu'à partir d'une surface libre au repos, les vagues ne sont générées que vers la droite du canal, ce qui est confirmé par nos simulations (Fig. 2).

Les simulations ont été réalisées dans un canal de longueur $L=20 \mathrm{~m}$ et de profondeur $d=3 \mathrm{~m}$, avec une plage absorbante sur les 7 derniers mètres. Un cylindre de rayon $R=0,1 \mathrm{~m}$ est placé à $5 \mathrm{~m}$ de la frontière gauche, à $z_{c}=-3 R$ sous la surface libre au repos. Le cylindre est alors mis en mouvement de façon progressive sur 4 périodes de rotation (afin d'éviter les instabilités liées à un démarrage impulsif) pour finalement se placer sur une orbite circulaire avec une vitesse angulaire $\omega$. L'hypothèse de profondeur infinie faite par $\mathrm{Wu}$ est bien vérifiée dans nos simulations avec $k d=5$. On a utilisé 200 nœuds sur la surface libre et 80 sur le corps. Les simulations durent 15 périodes de rotation.

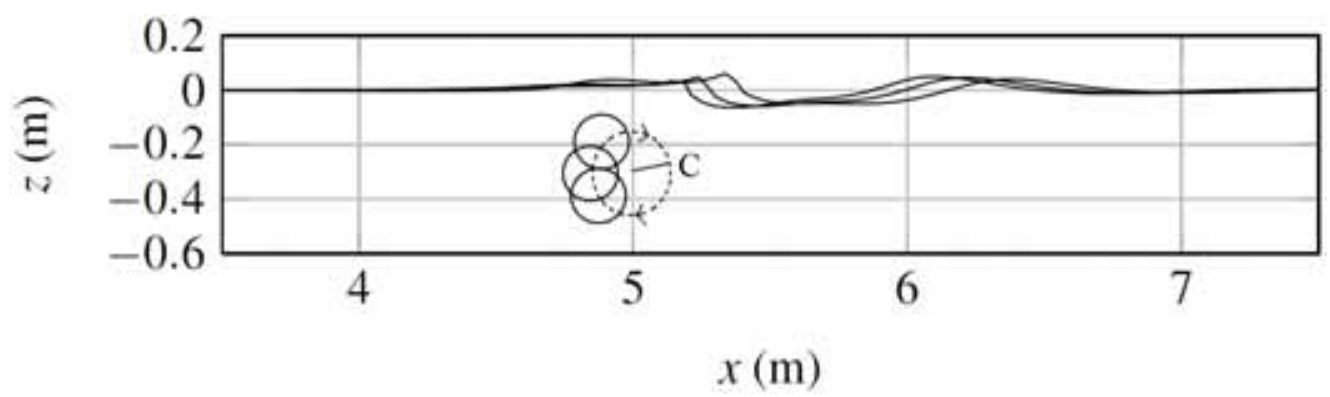

Figure 2. Position du cylindre et de la surface libre à 3 instants successifs $(t / T=4,40 ; t / T=4,49 ; t / T=4,62)$.

De façon similaire à $\mathrm{Wu}$, on décompose les efforts vertical et horizontal adimensionnés en séries de Fourier sous la forme :

$$
\left\{\begin{array}{l}
\frac{F_{x}}{C \rho \pi R^{2} \omega^{2}}=F_{x}^{(0)}+\sum_{n \geq 1} F_{x}^{(n)} \cos \left(n \omega t+\varphi_{n}\right) \\
\frac{F_{z}}{C \rho \pi R^{2} \omega^{2}}=F_{z}^{(0)}+\sum_{n \geq 1} F_{z}^{(n)} \sin \left(n \omega t+\varphi_{n}\right)
\end{array}\right.
$$

Les figures $3 \mathrm{a}, 3 \mathrm{~b}$ et $3 \mathrm{c}$ comparent la composante moyenne ainsi que les amplitudes des deux premiers harmoniques des forces adimensionnées aux résultats de WU (1993) pour les 8 amplitudes de mouvement. 


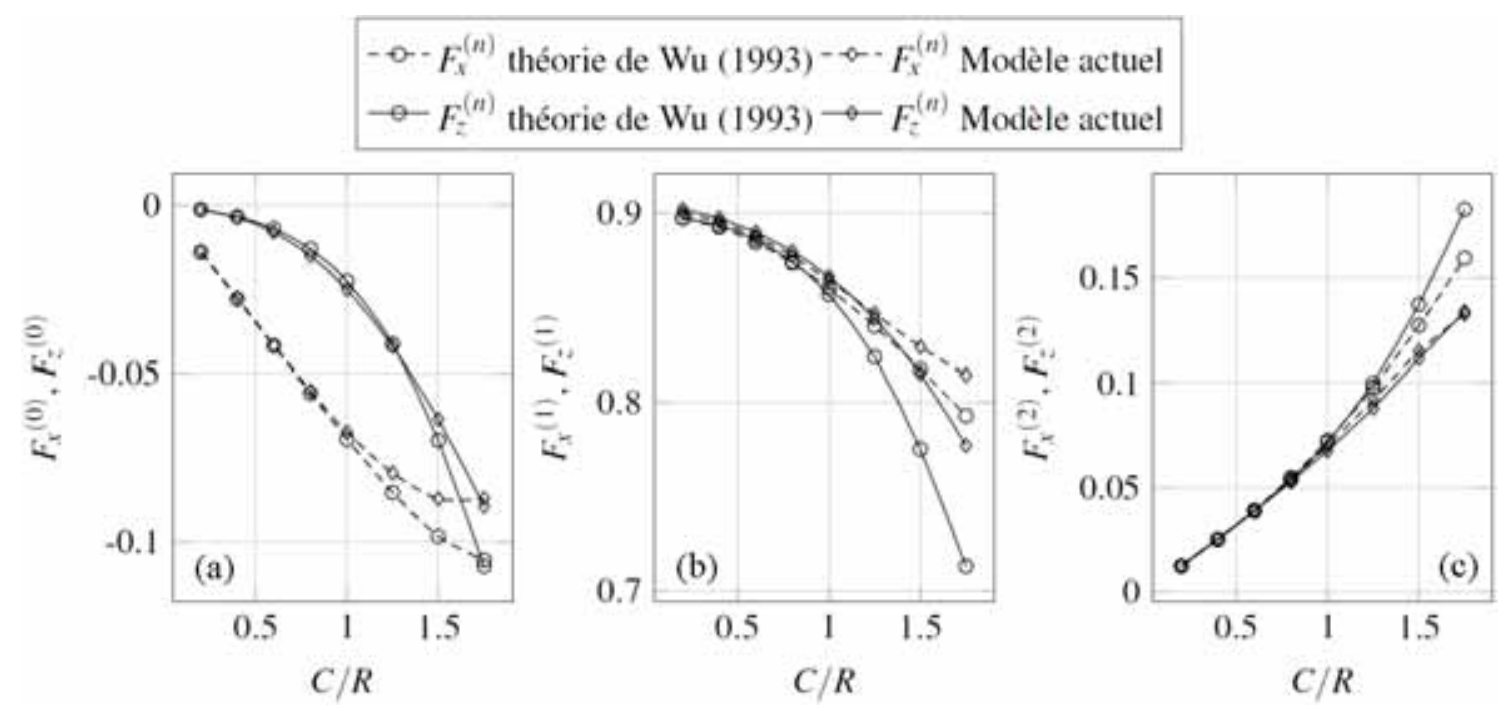

Figure 3. Moyenne (a), amplitude du $1^{\text {er }}$ harmonique (b) et du $2^{\text {nd }}$ harmonique (c) des forces horizontale et verticale adimensionnées en fonction du rayon orbital adimensionné $C / R$, comparés aux résultats de la théorie de WU (1993).

Ces figures montrent un bon accord avec la théorie de WU (1993) pour les amplitudes relativement faibles $(C / R<1)$. Au-delà le cylindre se rapproche de façon significative de la surface libre et les effets non-linéaires ne sont alors plus négligeables, comme le montre la figure 4 sur les forces verticale et horizontale simulées.

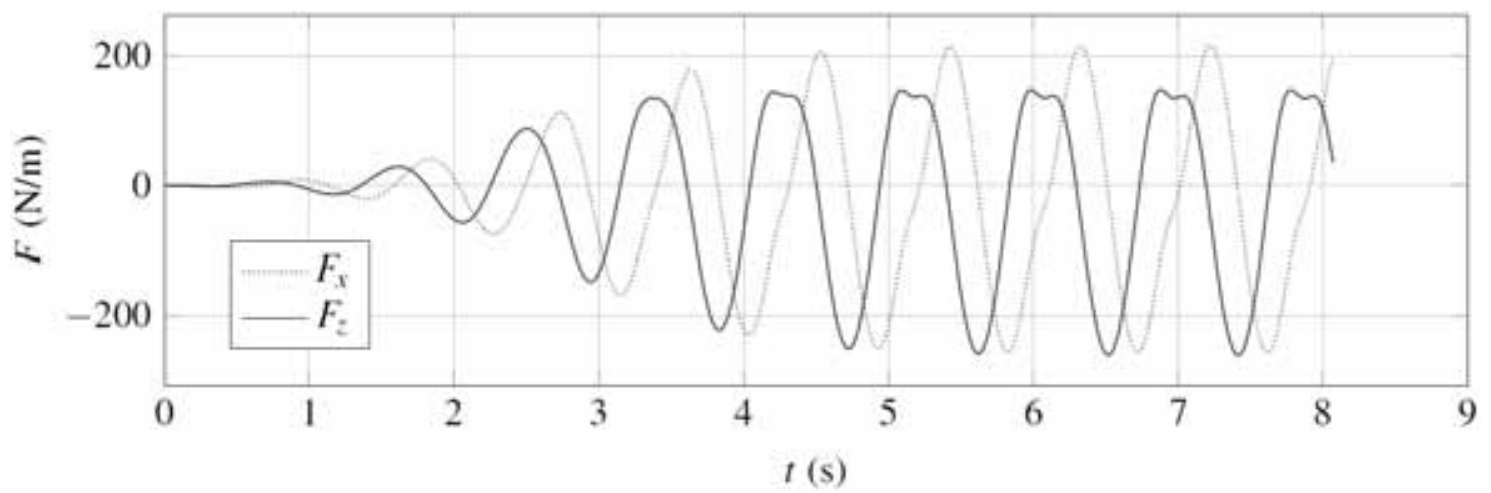

Figure 4. Forces verticale et horizontale simulées, $k R=0,5, C / R=1,75$.

Des résultats obtenus par la méthode HOS (KENT \& CHOI, 2007) ont également été comparés à ceux de $\mathrm{Wu}$, malheureusement pour des rayons orbitaux inférieurs à $C / R=0,6$, amplitude à laquelle les effets non-linéaires n'apparaissent pas clairement.

\section{Cas du cylindre en mouvement "libre"}

Nous avons vu précédemment qu'un cylindre immergé décrivant un mouvement orbital circulaire se comporte comme un générateur de vagues unidirectionnel. Inversement, il est possible d'imaginer qu'un cylindre immergé placé dans un champ de vagues et 
convenablement ancré au fond ou à une structure fixe par un système de ressorts et d'amortisseurs pourrait absorber efficacement l'énergie des vagues, et potentiellement constituer un SREV. Cette idée a été introduite et étudiée dans les années 1980, via le "Cylindre de Bristol" (EVANS et al., 1979). La théorie au $1^{\mathrm{er}}$ ordre (linéaire) développée par Evans et al. sert de point de comparaison à nos simulations. On considère ici le mouvement d'un cylindre immergé sous l'action de vagues périodiques. L'équation du mouvement d'un tel cylindre de masse $M$ par unité de longueur, à la position $\underline{x}$ à l'instant $t$ partant d'une position d'équilibre $\underline{X}_{i n i}$ s'écrit dans ce cadre :

$M \underline{\ddot{x}}=\underline{F}_{h}+M \underline{g}-d_{0} \underline{\dot{x}}-k_{0}\left(\underline{x}-\underline{x}_{\text {ini }}\right)$

$\underline{F}_{h}$ représente les efforts hydrodynamiques induits par les vagues et $\left(k_{0}, d_{0}\right)$ sont respectivement la raideur et le coefficient d'amortissement, pris égaux dans les directions $x$ et $z$, et calculés en fonction d'une pulsation de réglage $\omega_{0}=2 \pi f_{0}$, pour laquelle la puissance absorbée est maximale (EVANS et al., 1979) :

$\left\{\begin{array}{l}k_{0}=\left(M+a_{i i}\left(\omega_{0}\right)\right) \omega_{0}^{2} \\ d_{0}=b_{i i}\left(\omega_{0}\right)\end{array}\right.$

où $a_{i i}\left(\omega_{0}\right)$ et $b_{i i}\left(\omega_{0}\right)$ sont respectivement la masse ajoutée (linéaire) et le coefficient d'amortissement de radiation du cylindre, à la fréquence de réglage. EVANS et al. (1979) montrent que, sous ces conditions, le cylindre décrit un cercle de rayon $C$ sous l'action d'une houle linéaire d'amplitude $A$ et de pulsation $\omega$ :

$$
\left(\frac{C}{A}\right)^{2}=\frac{\rho g^{2} b_{i i}(\omega)}{\omega^{3}\left\{\left(d_{0}+b_{i i}(\omega)\right)^{2}+\frac{1}{\omega^{2}}\left[k_{0}-\left(M+a_{i i}(\omega)\right) \omega^{2}\right]^{2}\right\}}
$$

La configuration utilisée par Evans et al. est reproduite dans nos simulations : un cylindre de rayon $R=0,05 \mathrm{~m}$, réglé à la fréquence $f_{0}=1,65 \mathrm{~Hz}$, est placé à la cote initiale $z_{c}=-0,0625 \mathrm{~m}$ sous la surface libre au repos dans un canal de longueur $20 \mathrm{~m}$ et de profondeur $0,60 \mathrm{~m}$, avec une plage absorbante sur les 7 derniers mètres. Des vagues monochromatiques de faible amplitude $(A / R=0,0033)$ sont générées avec 8 fréquences différentes réparties entre $1 \mathrm{~Hz}$ et $2 \mathrm{~Hz}$.

Après un état transitoire, le centre du cylindre décrit une trajectoire quasi-circulaire, stable dans le temps, représentée sur la figure $5 \mathrm{a}$ (cas $f=f_{0}$, soit $k R=0,55$ ). Ce type de trajectoire est observé pour les 8 fréquences considérées. La figure $5 \mathrm{~b}$ montre l'évolution du rayon de la trajectoire $C$ (adimensionné par le rayon du cylindre $R$ ) en fonction de $k R$, en comparaison à la théorie linéaire d'EVANS et al. (1979). Le rayon calculé dans les simulations numériques est légèrement plus faible que le rayon prédit par la théorie linéaire, ce qui est sans doute lié à des effets non-linéaires (en cours d'analyse actuellement). 


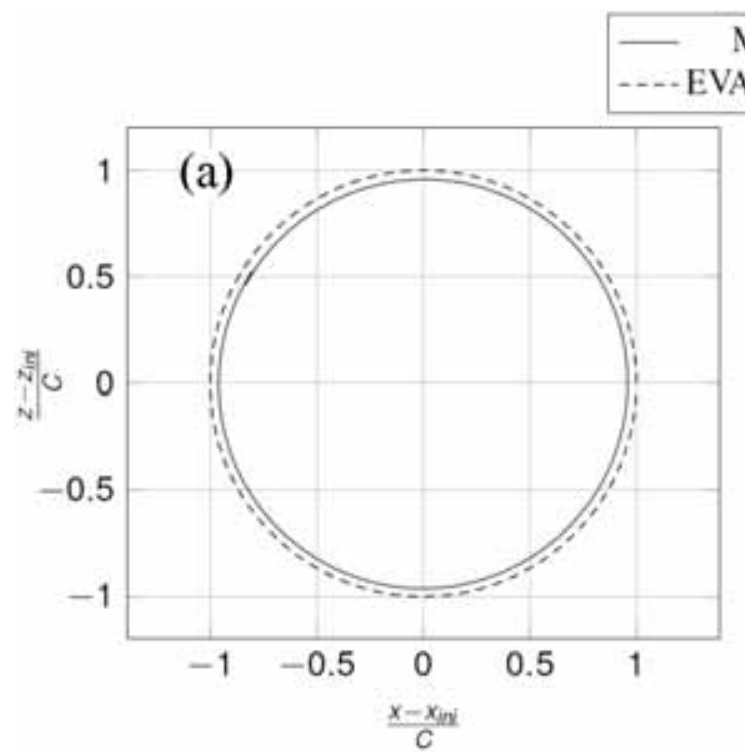

Modèle actuel

EVANS et al. (1979)

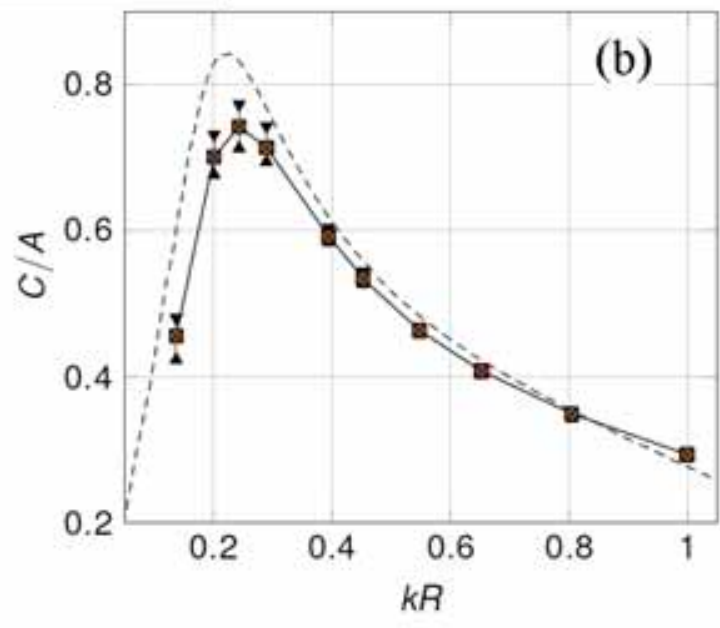

Figure 5. (a) Trajectoire du centre d'inertie du cylindre réglé à la fréquence $f_{0}=1,65 \mathrm{~Hz}$ sur une période de vague (cas $f=f_{0}$, soit $k R=0,55$ )

(b) Evolution du rayon de la trajectoire pour différentes fréquences de vague.

La fréquence de réglage correspond à $k R=0,55$. Les symboles font référence aux rayons minimum, moyen et maximum simulés sur une période de vague.

Ce premier cas-test confirme la capacité du modèle à simuler la dynamique d'un corps "libre" soumis à l'action des vagues. Il s'agit également d'une première application du modèle à un cas réaliste, en vue de modéliser la dynamique de SREV immergés.

\section{Conclusions et perspectives de développement}

Le modèle numérique utilisé, à savoir un canal à houle numérique bidimensionnel, fondé sur un modèle potentiel complètement non-linéaire, a été présenté. Le problème du couplage hydro-mécanique, lié à la présence d'un corps cylindrique totalement immergé en mouvement imposé ou "libre", a été formulé de façon mathématique et résolu de façon numérique par la méthode implicite de VAN DAALEN (1993) et TANIZAWA (1995). Les résultats du modèle ont ensuite été comparés à la théorie de WU (1993) pour le cas d'un cylindre en mouvement imposé, et à la théorie linéaire d'EVANS et al. (1979) pour le cas d'un cylindre en mouvement "libre" (avec ressort et amortissement dans les directions horizontale et verticale), représentant un SREV idéalisé.

Les travaux actuels concernent l'extension du modèle pour simuler des états de mer irréguliers (définis par un spectre de variance donné), l'examen de la possibilité de tenir compte des effets visqueux à l'aide d'un terme de force supplémentaire, et le traitement de cas de SREV plus réalistes, avant de procéder ensuite à une extension du modèle à la simulation du problème $3 \mathrm{D}$. 
Modélisation non-linéaire des interactions des vagues avec un corps mobile immergé : 2.11

\section{Références bibliographiques}

CAO Y., BECK R.F., SCHULTZ W.W. (1994). Nonlinear Computation of Wave Loads and Motions of Floating Bodies in Incident Waves. Proc. $9^{\text {th }}$ Int. Workshop Water Waves Floating Bodies, Kuju, Oita, Japan.

CHAPLIN J.R. (1984). Nonlinear Forces on a Horizontal Cylinder Beneath Waves. J. Fluid Mech., 147, pp 449-464. doi:10.1017/S0022112084002160

COINTE R. (1989). Quelques aspects de la simulation numérique d'un canal à houle. Thèse de l'Ecole Nationale des Ponts et Chaussées, 284 p.

EVANS D.V., JEFFREY D.C., SALTER S.H., TAYLOR J.R.M. (1979). Submerged cylinder wave energy device: theory and experiment. Applied Ocean Res., 1(1), pp 3-12. doi:10.1016/0141-1187(79)90003-8

GRILLI S.T. (1997). Fully Nonlinear Potential Flow Models used for Long Wave Runup Prediction: Chapter in "Long-Wave Runup Models". World Scientific Publishing, Singapore, pp 116-180.

GRILLI S.T., HORRILLO J. (1997). Numerical Generation and Absorption of Fully Nonlinear Periodic Waves. J. Eng. Mech., 123(10), pp 1060-1069. doi:10.1061/(ASCE)07339399(1997)123:10(1060)

GRILLI S.T., SUBRAMANYA R. (1996). Numerical Modeling of Wave Breaking Induced by Fixed or Moving Boundaries. Comp. Mech., 17(6), pp 374-391. doi:10.1007/BF00363981

KENT C.P., CHOI W. (2007). An Explicit Formulation for the Evolution of Nonlinear Surface Waves Interacting with a Submerged Body. Int. J. Numer. Meth. Fluids, 55, pp 1019-1038. doi:10.1002/fld.1504

MANN L.D., BURNS A.R., OTTAVIANO M.E. (2007). CETO: a Carbon Free Wave Power Energy Provider of the Future. Proc. $7^{\text {th }}$ European Wave and Tidal Energy Conference, Porto, Portugal.

SEN D. (1993). Numerical Simulation of Motions of Two-Dimensional Floating Bodies. J. Ship Res., 37(4), pp 307-330.

TANIZAWA K. (1995). A Nonlinear Simulation Method of 3D Body Motions in Waves, $1^{\text {st }}$ Report. J. Soc. Nav. Arch. Japan, 178, pp 179-191.

VAN DAALEN E.F.G. (1993). Numerical and Theoretical Studies of Water Waves and Floating Bodies. PhD Thesis, University of Twente, The Netherlands, 267 p.

VINJE T., BREVIG P. (1981). Nonlinear, Two-Dimensional Ship Motions. Norwegian Institute of Technology, Report R-112.81.

WU G.X. (1993). Hydrodynamic Forces on a Submerged Circular Cylinder Undergoing Large-Amplitude Motion. J. Fluid Mech., 254, pp 41-58. doi:10.1017/S0022112093002022 
2.12 : Revue Paralia - Vol. 4 (2011)

WU G.X., EATOCK-TAYLOR R. (1996). Transient Motion of a Floating Body in Steep Water Waves. Proc. $11^{\text {th }}$ Int. Workshop Water Waves Floating Bodies, Hamburg, Germany. 